\title{
Integration of GNSS and INS with a phased array antenna
}

\author{
Saeed Daneshmand ${ }^{1} \cdot$ Gérard Lachapelle $^{1}$
}

Received: 16 May 2017 / Accepted: 13 October 2017 / Published online: 4 November 2017

(C) The Author(s) 2017. This article is an open access publication

\begin{abstract}
High attenuation, blockage and severe multipath fading in urban environments, under dense canopy or jamming attacks degrade accuracy, continuity, availability and integrity of GNSS services. GNSS/INS integration and antenna array beamforming approaches both provide certain levels of protection against these challenging circumstances in different ways and are studied in the literature; however, their combination of them has received less attention. This research studies different strategies to combine a GNSS antenna array with an inertial navigation system. The focus is on the integration of ultra-tight and tightly coupled GNSS/ INS with a distortionless GNSS beamformer. It is shown that a tighter integration of a phase array antenna with INS and GNSS not only has all the benefits of array processing and INS in dealing with challenging environments, but also can provide external information for attitude parameters, and therefore, the overall performance of the integrated system is improved. To verify the applicability of the integrated system and to evaluate its performance, two sets of data have been collected and analyzed.
\end{abstract}

Keywords Array processing - Blind and distortionless beamforming · GNSS/INS integration · Interference suppression

Saeed Daneshmand

sa.daneshmand@gmail.com

Gérard Lachapelle

gerard.lachapelle@ucalgary.ca

1 Department of Geomatics Engineering, PLAN Group

Schulich School of Engineering, University of Calgary, 2500

University Drive, N.W., Calgary, AB T2N 1N4, Canada

\section{Introduction}

Despite the recent advances in global navigation satellite system (GNSS) technology, jamming, blockage and severe multipath fading can still significantly impair the performance of receivers or completely deny GNSS positions and time services. Interference can impact the front end, acquisition, tracking and position stages of a receiver (Borio et al. 2016). Even a low-power jamming signal can potentially jeopardize the functionality of receivers in a circular region around the jammer with a radius of several kilometers. Low-cost jammers commonly known as personal privacy devices (PPD) can be legally or illegally procured although laws prohibit usage of such devices. Moreover, due to everincreasing built-up urban environments where most satellite signals are faded or blocked, pure GNSS-based positioning and navigation becomes inaccurate and unreliable. Antenna array processing and GNSS/inertial navigation system (INS) integration are two highly effective approaches to combat these challenging environments.

Processing techniques utilizing an array of antennas can effectively defeat various types of GNSS interference and jamming signals regardless of their temporal or spectral characteristics (Fernández-Prades and Arribas 2016; Daneshmand et al. 2016; Cuntz et al. 2016). As long as the size, cost and power consumption of additional front ends and antennas are justifiable, antenna array processing is one of the most powerful countermeasures against both narrowand wideband interfering signals. In urban environments, an antenna array-based receiver can alleviate multipath effects and increase the $\mathrm{C} / \mathrm{N}_{0}$ by steering the main lobe of the array gain pattern toward the satellite signal directions (Seco-Granados et al. 2003; Fante and Vaccaro 2000; Daneshmand et al. 2013). However, the array-based advantage depends 
on the availability of the GNSS signals and degrades under blockage and outage.

Inertial navigation systems (INS) are self-contained navigation systems that provide position, velocity and attitude information by measuring and integrating acceleration and angular velocity. An INS provides this information by using an inertial measurement unit (IMU) and applying methods such as dead reckoning (DR) (Noureldin et al. 2009). Given initial conditions and continuous acceleration and angular velocity measurements, one integration of acceleration provides velocity and a second integration, along with attitude parameters, gives 3D position. Angular rates are processed to provide the attitude parameters in the form of pitch, roll and yaw angles and also to transform navigation parameters from the body frame to the local-level frame coordinate system. Although an INS is immune to interference and multipath, it cannot provide long-term high-accuracy position solutions (Grewal et al. 2007; Wong et al. 1988). The inherent drifts and biases in IMU measurements, if not correctly dealt with, or aided as part of an integrated solution, can cause significant error over time. Among sources that can provide reliable external aid for an INS, GNSS is of great interest due to its low user equipment cost and global availability. Compared to INS, GNSS has less short-term accuracy but can provide higher long-term position accuracy (Noureldin et al. 2013). Therefore, due to this complementary nature of both systems, GNSS/INS integrated solutions are widely used in many applications (Godha et al. 2006; Petovello et al. 2009). A GNSS/INS solution combines IMU and GNSS measurements. The combined solution benefits from the strengths of both systems. Integrating antenna array-based GNSS with INS can even provide stronger protection against harsh environments, which is the focus of this research.

The use of antenna arrays in GNSS applications is growing rapidly, especially due to their superior capability to suppress both narrowband and wideband interference. Owing to the rapid advances and miniaturization of electronic systems and antenna design technologies, the hardware complexity of antenna array-based receivers is becoming significantly less challenging. An array either in a form of a multi-antenna receiver or a complex stand-alone antenna can be used in many GNSS applications. The idea of employing an array of antennas along with an IMU to provide attitude parameters has been thought of before and used in practice, especially in military applications. In beamforming approaches such as minimum variance distortionless response (MVDR), the knowledge of satellite steering vectors and consequently the array platform attitude parameters is required. Employing IMUs, if not the only practical solution, is certainly one of the best ones to provide attitude parameters especially in dynamic situations (De Lorenzo et al. 2005; Backen et al. 2008; Li et al. 2014; Brown and Matthews 2006; Li et al.
2011). That being said, albeit the apparent advantage of combining special processing and INS/GNSS integration, limited literature has been published to discuss implementation details of such combined systems. One reason is that in most studies the focus has been on array processing rather than the navigation system and the receiver design. Therefore, the integration of INS with an antenna array is either not discussed or attitude parameters are considered known a priori. Another reason is that a complete implementation and development of such systems is likely the scope of military applications and details are not publicly available.

We briefly review different types of GNSS/INS integration, namely loosely, tightly and ultra-tightly coupled, and two GNSS beamformers, namely blind and distortionless. Possible integration strategies for array GNSS with INS and related advantages and disadvantages of each are discussed. The especial focus is on ultra-tight and tightly coupled GNSS/INS/distortionless beamforming. To compare the performance of the proposed approach with the conventional implementation, actual GNSS and IMU data and a combination of hardware and software simulators are used to simulate a jamming attack situation. A multi-antenna software receiver is employed to analyze the performance suggested approaches.

Herein, the following notation is adopted: Small letters with arrow stand for vectors, and capital bold letters stand for matrices. Superscripts $\mathrm{H}$ and $\mathrm{T}$ denote conjugate transpose and transpose, respectively.

\section{INS and single-antenna GNSS integration}

INS, by using the mechanization equations, converts the output of IMU raw data into high-rate position, velocity and attitude information. In one form of these equations, the attitude parameters are first estimated from the gyroscope triad considering the effect of earth rotation. After compensating for gravity, the accelerometer triad measurements are projected to the local-frame coordinate system and integrated to obtain 3D velocity and finally further integrated to obtain $3 \mathrm{D}$ position. INS errors grow over time owing to the implicit mathematical integrations in the mechanization algorithm, which cause bias errors of accelerometers and gyroscopes to accumulate at the sensor outputs. The bias errors of the inertial sensors are known as long-term errors. On the contrary, GNSS is prone to short-term noise, but it can provide better long-term position and velocity estimates. Depending on the application and compromises between simplicity versus performance, there are different types of GNSS/INS integration. In the following, three well-known architectures of GNSS/ INS integration, namely loosely coupled, tightly coupled and ultra-tight (deeply coupled), are introduced. 


\section{Different types of GNSS/INS integration}

In a loosely coupled integration, the GNSS and INS processes provide independent navigation solutions. An optimal estimator such as a Kalman filter then combines the solutions to achieve better performance. The distinctive feature of this implementation is applying separate filters for the GNSS and INS in addition to a combing filter. INS errors are estimated and compensated using GNSS measurements to avoid long-term biases and drifts. The main advantage of this implementation is simplicity, and its main drawback is the lack of GNSS aiding and integration when the effective number of satellites falls below the minimum number (four for the general case) of satellites needed to calculate the time and position solutions. This limits the applicability of this approach in challenging environments.

In a tightly coupled integration, the measurements from the GNSS and INS are integrated at a deeper level. Contrary to the loosely coupled integration, there is only one central filter. In this architecture, pseudorange and pseudorange rate measurements or correlator output's in-phase and quadrature components from the GNSS receiver, and accelerations and angular rates measurements from IMUs are combined in one filter, generally a Kalman filter (Noureldin et al. 2013; Groves 2008). Therefore, this integration requires access to the receiver's internal process. Tightly coupled integration eases the constraint on the minimum number of satellites for the GNSS process, and any number of satellites can still provide information. At the cost of increasing complexity, it outperforms the loosely coupled architecture, especially in harsh environments.

Ultra-tight or deep integration takes advantage of the fact that the GNSS signals are related via the receiver's position and velocity in order to aid weaker signal tracking and acquisition and overall to increase the signal-to-noise ratio. The ultra-tight and tightly coupled integrations are similar in terms of integration approach and related equations. The difference is in the GNSS receiver structure in ultra-tight to improve the tracking performance by employing the vectorbased (VB) tracking methodology (Petovello et al. 2008a, b). Due to its capability to track weaker signals in harsh environments, VB is now of great interest. Its receiver structure, however, is much more complicated. Instead of a conventional pair of delay-locked loop (DLL) and frequency-locked loop (FLL) for each channel, a navigation filter performs code phase and carrier frequency tracking for all PRNs. Replacing phase-locked loop (PLL) is not, however, straightforward and practical. The effects of different ionospheric errors on each satellite signal and satellite oscillator errors make it very hard to track phase from the navigation solution feedback loop (Petovello et al. 2008a, b). In one implementation of VB tracking, the code phase and the carrier frequency of the numerical controlled oscillator (NCO) are controlled by the navigation solution filter, while the carrier phase tracking is controlled in each channel individually. Extending the VB receiver to ultra-tight by following the same concept of tightly coupled strategy is straightforward (Petovello and Lachapelle 2006). Ultra-tight integration is simply a VB receiver integrated with an IMU in a tightly coupled manner. The main difference between them is that ultra-tight integration utilizes a GNSS/INS integrated navigation solution rather than a GNSS-only navigation solution in the case of VB receiver. Another advantage of ultra-tight integration compared to loosely and tightly coupled integration is the possibility of having a narrower tracking loop filter and a longer coherent integration time since with highrate IMU measurements the dynamics of the user motion can be removed in the tracking filter.

\section{System and measurement model for the integration filter}

There are several methods for optimal integration of GNSS and INS data such as Kalman filtering, particle filtering and artificial intelligence approaches. Among them, the Kalman filter and its various forms are employed mostly in practice. Kalman filtering utilizes the system's dynamic and the measurements to predict the system's behavior over time and estimate the parameters of interest in the system states. In order to use this filter, the system should be linear or linearized using either predefined information or the previous best estimate of the system states. Kalman filtering considers the accuracy of the input measurements by appropriately weighting them in the optimization algorithm. As mentioned, IMU errors grow over time and need to be estimated and compensated. From regular measurement updates, Kalman filter state errors are fed back to the inertial system to correct gyro-drifts and accelerometer biases in the long term and the INS provides short-term, accurate and high-rate system state estimates which in turn preserve the linearity assumption required for the optimization. It is more convenient to design the filter based on states errors rather than states themselves. INS errors such as initial misalignment, accelerometer and gyro-biases are estimated and compensated in the Kalman filter over time using external information from GNSS measurements. The following system and measurement models are applicable for tightly coupled and ultra-tight integration. These models are extended to GNSS INS \phased array integration in "Antenna array GNSS and INS integration" section. The discrete form of the GNSS/ INS system model can be written as

$\delta \vec{x}_{k}=\mathbf{F} \delta \vec{x}_{k-1}+\vec{w}_{k-1}$

where $\delta \vec{x}$ is the state error vector that typically consists of position $(\delta \vec{r})$, velocity $(\delta \vec{v})$ and attitude $(\delta \vec{\varepsilon})$ errors as well as 
gyroscope drifts $(\delta \vec{\omega})$, accelerometer biases $(\overrightarrow{\delta f})$ and finally the receiver clock error $\left(\delta b_{r}\right)$ and its drift $\left(\delta b_{d}\right)$ as

$\delta \vec{x}=\left[\begin{array}{ccccccc}\delta \vec{r}^{\mathrm{T}} & \delta \vec{v}^{\mathrm{T}} & \delta \vec{\varepsilon}^{\mathrm{T}} & \delta \vec{\omega}^{\mathrm{T}} & \delta \vec{f}^{\mathrm{T}} & \delta b_{r} & \delta b_{d} \\ 3 \times 1 & 3 \times 1 & 3 \times 1 & 3 \times 1 & 3 \times 1 & \end{array}\right]^{\mathrm{T}}$

For simplicity, the gyro- and accelerometer scale factors and other minor sources of error are not considered in the problem formulization. In (1), $\vec{w}$ is a white Gaussian noise vector and $\mathbf{F}$ is the dynamic coefficient matrix consisting of INS sensor and GNSS receiver clock models.

The measurement model for the Kalman filter in the discrete-time domain is expressed as (Noureldin et al. 2013) optimization problem. In order to calibrate an antenna array, a common approach is to use an anechoic chamber to scan all incident signals from different directions (Kim et al. 2004). For GNSS applications, the satellite signals themselves can be used as signal sources with known positions for the calibration process. By employing these signals along with an IMU to provide attitude parameters of the array platform, the calibration can be done. This approach for array calibration is referred to as on-site calibration (Backen et al. 2008; Daneshmand et al. 2014a, b). After calibration and during the normal operation, an IMU is still required to provide attitude parameters for distortionless beamforming (De Lorenzo et al. 2005; Li et al.

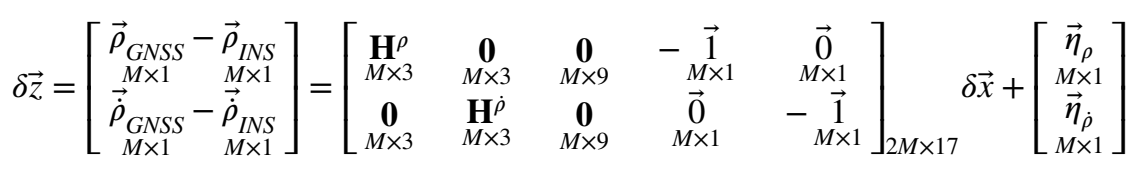

where $\delta \vec{z}$ is the differential observation vector and $\vec{\rho}$ and $\vec{\rho}$ are pseudorange and pseudorange rate measurement vectors obtained from GNSS signals and $\mathbf{H}^{\rho}$ and $\mathbf{H}^{\dot{\rho}}$ are corresponding design matrices. The differential observation vector is the difference between pseudorange and pseudorange rate measurements predicted by the INS and the ones measured by the receiver. $M$ in (3) is the number of observations or the number of satellites. From the satellite navigation message, and other predefined models, atmospheric errors, satellite position and clock error and other error sources should be calculated and considered when pseudorange and pseudorange rate measurement vectors are differentiated in the equation. Before describing how to integrate the phased array into these equations, different types of GNSS beamforming are reviewed in the following section.

\section{GNSS beamforming}

GNSS beamformers can be categorized into two general groups. In the first group, the beamformer is designed to maximize the received power for satellite signals and minimize the received power for undesired signals as in the MVDR or minimum power distortionless response (MPDR) methods (Van Trees 2002; Arribas and Fernandez-Prades 2013). Generally, in this type of beamforming, the optimization is performed for each satellite signal separately by using the signal direction of arrival and the array platform attitude parameters information. In this method, the antenna array should be fully calibrated since the signal's spatial phase information is employed in the constrained optimization. Here, these beamformers are referred to as distortionless since there is no distortion on the carrier phase measurements as it is compensated in the
2014; Brown and Matthews 2006; Li et al. 2011).

The second type of GNSS beamformers, known as blind beamformers, is much simpler in terms of complexity and implementation. In this case, the desired signals in the optimization are disregarded. Assuming that the power values of undesired signals are much higher, the received power is minimized. Since the spatial information of the desired signals in the optimization is disregarded, it has the drawback of possible loss or attenuation of some satellite signals (Zoltowski and Gecan 1995; Reed and Yao 1998; Carrie et al. 2005). However, due to its simplicity, this method is also of great interest since the array processing functionality can be fully performed before Doppler removal and correlation stages of a receiver and it can be implemented independent of the receiver's structure, and therefore, it can be used with off-the-shelf GNSS receivers. In the following subsections, first a system model for signals received at an antenna array is presented, and then, blind and distortionless beamformers are reviewed.

\section{System model}

Assume that an $\mathrm{N}$-element antenna array with an arbitrary configuration is placed on a platform that is fixed with respect to the body frame coordinate system. The $N \times 1$ vector of complex baseband signals consisting of measured phases and amplitudes at different antennas for one satellite signal can be written as

$\vec{y}=\mathbf{C} \vec{a}+\vec{\eta}$

where $\vec{a}$ is an $N \times 1$ vector representing the steering vector (array manifold vector) of the satellite signal defined as 
$\vec{a}=\left[\begin{array}{c}e^{j \frac{2 \pi}{\lambda} \vec{z}_{1}^{T} \vec{e}^{B}} \\ e^{j \frac{2 \pi}{\lambda} \vec{z}_{2}^{T} \vec{e}^{B}} \\ \vdots \\ e^{j \frac{2 \pi}{\lambda} \vec{z}_{N}^{T} \vec{e}^{B}}\end{array}\right]$

in which $\lambda$ is the wavelength of the signal and $\vec{z}_{n}, n=1,2, \ldots, N$ is a $3 \times 1$ vector pointing to the $n$th antenna element and $\vec{e}^{B}$ is a $3 \times 1$ unit vector pointing to the satellite in the body frame coordinate system. In (4), $\vec{\eta}$ is a complex Gaussian noise vector and $\mathbf{C}$ is a $N \times N$ matrix modeling constant uncertainties due to antenna imperfections such as unequal cable lengths and coupling coefficients between antenna elements. The steering vector conveys the attitude information of the platform on which the array is mounted on. $\vec{e}^{B}$ can be stated as a function of attitude parameters and satellite azimuth and elevation angles as (Konovaltsev et al. 2013; Daneshmand et al. 2013)

$\vec{e}^{B}=\mathbf{Q} \vec{e}^{E N U}=\mathbf{Q}\left[\begin{array}{c}\sin \left(\varphi^{E N U}\right) \cos \left(\lambda^{E N U}\right) \\ \cos \left(\varphi^{E N U}\right) \cos \left(\lambda^{E N U}\right) \\ \sin \left(\lambda^{E N U}\right)\end{array}\right]$

where $\mathbf{Q}$ is the transformation matrix from the east, north, up (ENU) coordinate system to the body frame coordinate system and $\vec{e}^{E N U}$ is a $3 \times 1$ unit vector pointing to the satellite in the ENU coordinate system. $\mathbf{Q}$ is formed based on three angles, namely $r, p$ and $y$, which refer to the roll, pitch and yaw (heading) angles and can be expressed as
(Van Trees 2002; Sgammini et al. 2012). The main advantage of blind beamforms is their low implementation complexity since the spatial information of satellite signals is not utilized in the optimization problem and the goal is to only nullify the high power interfering signals. The optimization problem for power minimization beamformer can be written as

$$
\begin{array}{r}
\underset{\vec{w}}{\operatorname{Min}} \vec{w}^{H} \mathbf{R} \vec{w} \\
\vec{c}^{H} \vec{w}=1
\end{array}
$$

where $\vec{w}$ is the array gain vector, $\mathbf{R}$ is the spatial correlation matrix and $\vec{c}$ is a constraint vector defined as

$$
\underset{N \times 1}{\vec{c}}=\left[\begin{array}{llll}
1 & 0 & \cdots & 0
\end{array}\right]^{\mathrm{T}}
$$

This constraint avoids the trivial solution of $\vec{w}=\overrightarrow{0}$. This minimization problem can be solved by using a Lagrange multiplier approach. The optimal gain vector is obtained as

$$
\vec{w}=\alpha \mathbf{R}^{-1} \vec{c}
$$

where $\alpha$ is a scale factor.

The optimization criteria for distortionless beamformer are the same as blind beamformer, but the constraint incorporates the satellite direction of the arrival information as

$$
\begin{gathered}
\underset{\vec{w}}{\operatorname{Min}} \vec{w}^{H} \mathbf{R} \vec{w} \\
\vec{a}^{H} \vec{w}=1
\end{gathered}
$$

where again, using a Lagrange multiplier approach, the optimal gain vector is obtained as

$$
\mathbf{Q}=\left[\begin{array}{ccc}
\cos (y) \cos (r)-\sin (r) \sin (p) \sin (y) & \sin (y) \cos (r)+\sin (r) \sin (p) \cos (y) & -\sin (r) \cos (p) \\
-\sin (y) \cos (p) & \cos (y) \cos (p) & \sin (p) \\
\cos (y) \sin (r)+\sin (y) \sin (p) \cos (r) & \sin (y) \sin (r)-\cos (y) \sin (p) \cos (r) & \cos (p) \cos (r)
\end{array}\right]
$$

Satellite azimuth and elevation angles in the ENU coordinate system, symbolized by $\varphi^{E N U}$ and $\lambda^{E N U}$ in (6), can be accurately estimated from the GNSS position solution. In "Antenna array GNSS and INS integration" section, it is shown how this information can be integrated into the GNSS/INS part to provide attitude information updates.

\section{Beamformers}

Blind beamforming is based on the fact that the power of interfering signals, in order to be destructive after correlation in the receiver, should be much higher than that of the pre-correlation GNSS signals, these being below the noise floor. The blind beamformer was first studied for GPS interference mitigation by Zoltowski and Gecan (1995) which was called power minimization beamformer. Another form of implementation is based on eigenvector decomposition

$$
\vec{w}=\alpha \mathbf{R}^{-1} \vec{a}
$$

The array gain vector for both beamforming approaches can also be obtained based on eigenvalue decomposition (EVD). In this approach, first a projection matrix to the interferencefree subspace from the spatial-temporal correlation matrix is calculated. Interference is destructive if its power is significantly higher than that of the GNSS and noise signals. This makes the interference subspace easily detected and estimated. A projection matrix into the interference-free subspace can be obtained by performing an EVD of $\mathbf{R}$ as

$$
\mathbf{R}=\left[\begin{array}{cc}
\mathbf{U}_{I n t} & \mathbf{U}_{N u l l} \\
N \times K & N \times(N-K)
\end{array}\right]\left[\begin{array}{cc}
\Lambda_{I n t} & \mathbf{0} \\
K \times K & \\
\mathbf{0} & \Lambda_{N u l l} \\
& (N-K) \times(N-K)
\end{array}\right]\left[\begin{array}{c}
\mathbf{U}_{\text {Int }}^{H} \\
\mathbf{U}_{\text {Null }}^{H}
\end{array}\right]
$$


where $\mathbf{U}_{\text {Int }}$ and $\mathbf{U}_{\text {Null }}$ are eigenvector matrices of interference and the noise-plus-GNSS signal subspaces, respectively, and $\Lambda_{\text {Int }}$ and $\Lambda_{\text {Null }}$ are corresponding eigenvalue matrices. In (13), $K$ indicates the rank of the interference subspace. $K$ is equal to or less than the number of interfering signals (equal when the interfering signals are uncorrelated). A projection matrix into the reduced-rank interference-free subspace can be calculated as $\mathbf{U}_{\text {Null }}^{H} \Lambda_{\text {Null }} \mathbf{U}_{\text {Null }}$, in which $\mathbf{U}_{\text {Null }}$ is formed from $N-K$ eigenvectors corresponding to the $N-K$ smallest eigenvalues. In fact, the filter gain vector $\vec{w}$ which minimizes the filter output power belongs to this interference-free subspace and can be expressed as

$\vec{w}=\alpha \mathbf{U}_{\text {Null }}^{H} \Lambda_{\text {Null }} \mathbf{U}_{\text {Null }} \vec{h}$

where $\vec{h}$ is an arbitrary unique vector. Considering the fact that $\mathbf{R}^{-1} \approx \mathbf{U}_{\text {Null }}^{H} \Lambda_{\text {Null }} \mathbf{U}_{\text {Null }}$ (Zoltowski and Gecan 1995), $\vec{w}$ is equivalent to the gain vectors obtained from power minimization and distortionless beamformers if $\vec{h}=\vec{c}$ and $\vec{h}=\vec{a}$, respectively. Eigen-beamformers result in sharper nulls for interfering signals (Citron and Kailath 1984); however, in contrast to the power minimization and distortionless beamformers, the interference dimension $K$ should also be determined to achieve the best performance.

\section{Antenna array GNSS and INS integration}

In this section, possible combinations of a phased array antenna, INS and GNSS are discussed. The combinations of these three range from systems working independently beside each other or deeply integrated together. The choice of a suitable and efficient receiver strategy is a function of operation environment and system dynamic as well as factors like performance and complexity. As stated before, the accuracy of an INS is affected by the sensor errors, initialization and computational algorithms. The situation is worse for the microelectromechanical system (MEMS) sensors where the INS output can drift rapidly due to severe errors. GNSS core prevents the inertial sensors from drifting, and INS provides continuity in the navigational solution. Processing signals from an array of antennas can increase the resistance of the receiver to interference, multipath and attenuation and also can provide attitude information. Attitude parameters estimated form spatial processing can provide extra information that helps to avoid inertial solution drift and therefore improves overall performance.

Depending on the beamforming approach and GNSS/INS integration, there are six possible architectures for GNSS/ INS/phased array integration. There are three combinations of blind beamforming with loosely, tightly and ultra-tight coupled GNSS/INS integrations and three combinations of distortionless beamforming with those three GNSS/INS integration types. From the previous discussion, a distortionless beamformer is much more complex and requires modifications in the receiver structure, while a blind beamformer is much easier to implement albeit with poorer performance. On the other hand, loosely coupled GNSS/INS is easy to implement, while tight and ultra-tight integrations are more complex but have better performance. These two require some connection between INS and GNSS or deep integration. Therefore, some of six possible array GNSS/INS integration architectures are more interesting, while others cannot be justified. For example, using a loosely coupled GNSS/INS integration with a complex distortionless multiantenna receiver while an inter-connection to the multiantenna from IMU is required anyway to provide attitude parameters for beamforming is not a good design. Similarly, it is a better choice to integrate ultra-tight GNSS/INS with a distortionless beamformer rather than a blind beamformer unless hardware considerations prevent this. In cases where the implementation simplicity is the most important objective, the combination of the blind beamformer with loosely coupled GNSS/INS is the best solution albeit with the poorest performance. In this case, the functionalities of GNSS, INS and array parts are independent. If the performance is the main objective, the combination of the distortionless beamformer with tightly coupled or ultra-tight GNSS/INS could be the best option.

It should be noted that due to the Schuler effect, the north and the east velocity component errors are bounded and also related to roll and pitch errors (Noureldin et al. 2013). Therefore, the velocity updates from GNSS receiver can estimate and compensate roll and pitch errors. However, there is no strong coupling with the yaw error which consequently grows over time especially in static cases. This in turn degrades the long-term accuracy of position and velocity estimates especially in harsh environments where GNSS may be regularly interrupted. The yaw error can also significantly affect the performance of the distortionless beamformer when the main lobe of beam pattern is not steered toward the direction of the satellite signal due to the attitude error especially when the number of antenna elements is large and the beam is narrow.

It is important to have a mechanism to update attitude parameters including the yaw angle. In addition to the superior anti-interference capability, antenna array processing can also be employed as an independent source of attitude error estimation and provide the attitude information from the estimated satellite steering vectors (Daneshmand et al. 2013). Contrary to velocity updates, all three attitude angle errors can be compensated even in a static case. This section focuses on how to integrate the attitude updates from array processing into ultra-tight or tightly coupled GNSS/ INS architectures. The Kalman equations are similar for both ultra-tight and tightly coupled implementations. The 


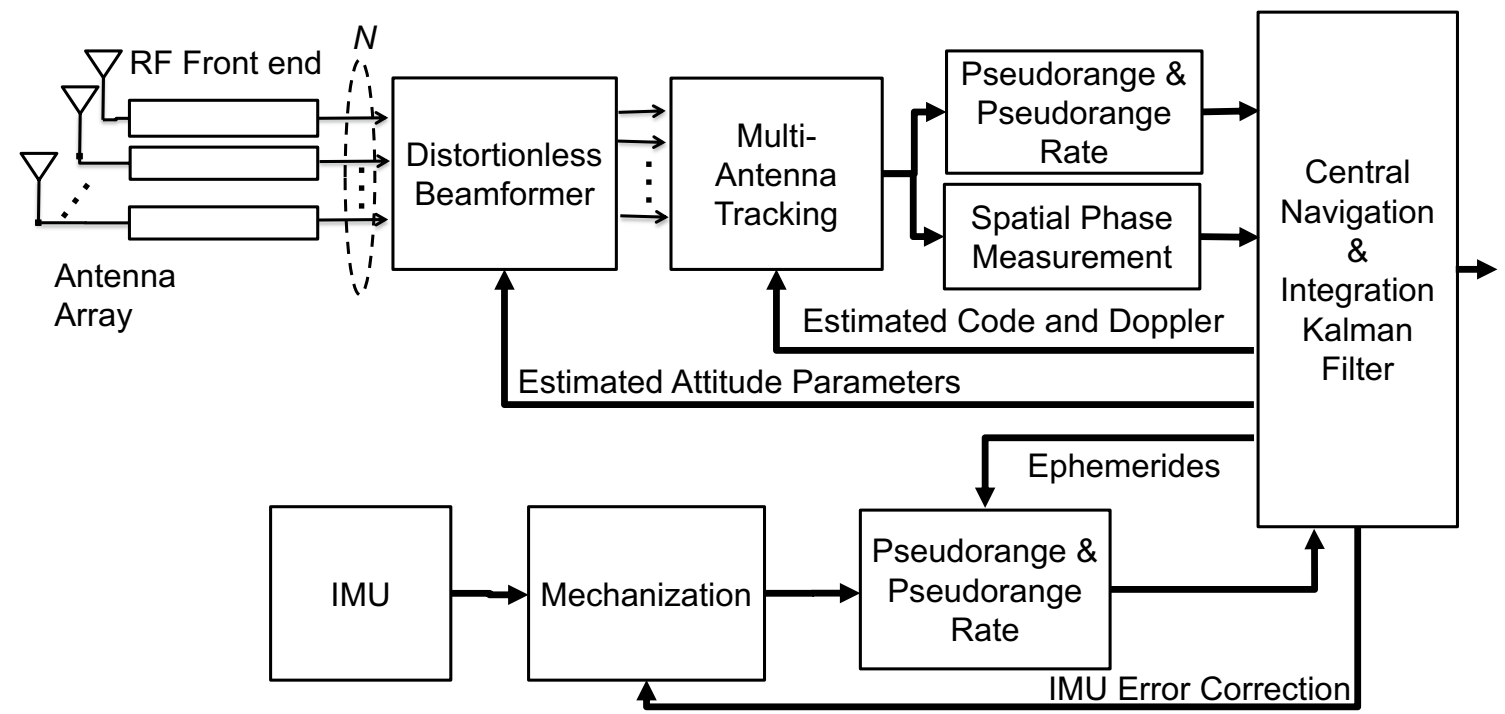

Fig. 1 General structure of ultra-tight GNSS/INS/distortionless beamformer integration

general schematic for ultra-tight GNSS/INS/distortionless beamformer integration is shown in Fig. 1. There is only one central Kalman filter. GNSS provides pseudorange and pseudorange rate measurement updates and array processing core in addition to beamforming provides spatial phase measurements. This structure suggests modifications only in the measurement model to incorporate the spatial phase measurements in the Kalman filter.

Considering (5), the measured phase vector can be obtained as

$\vec{\varphi}_{\text {GNSS }}=\frac{2 \pi}{\lambda} \mathbf{Z} \vec{e}^{B}$

in which $\mathbf{Z}$ is the $N \times 3$ array configuration matrix where its $n$th row is equal to $\vec{z}_{n}^{T}, n=1,2, \ldots, N$. As described in (6), by transferring the satellite vector into a local frame (ENU), $\vec{\varphi}_{G N S S}$ can be written as

$\vec{\varphi}_{G N S S}=\frac{2 \pi}{\lambda} \mathbf{Z} \mathbf{Q} \vec{e}^{E N U}$

where $\mathbf{Q}$ is defined in (7). Equation (16) is nonlinear with respect to the attitude parameters and should be linearized for the KF. As per (3), a first-order Taylor series expansion around $\vec{\varphi}_{I N S}$ (calculated directly from INS and estimated satellite azimuth and elevation angles) is obtained as

$\vec{\varphi}_{G N S S}=\vec{\varphi}_{I N S}+\frac{2 \pi}{\lambda} \mathbf{Z} \delta \mathbf{Q} \vec{e}^{E N U}+\frac{2 \pi}{\lambda} \mathbf{Z Q} \delta \vec{e}^{E N U}$

in which $\delta \mathbf{Q}$ is

$\delta \mathbf{Q}=\mathbf{Q}(p+\delta p, r+\delta r, y+\delta y)-\mathbf{Q}(p, r, y)$

If a rotation matrix is obtained as a result of two consecutive rotations with respect to the fixed reference frame, it is well known that the resulting rotation matrix can be described by the product of two rotation matrices as (Noureldin et al. 2013)

$\mathbf{Q}(p+\delta p, r+\delta r, y+\delta y)=\mathbf{Q}(p, r, y) \mathbf{Q}(\delta p, \delta r, \delta y)$

Therefore,

$\delta \mathbf{Q}==\mathbf{Q}(p, r, y) \mathbf{Q}(\delta p, \delta r, \delta y)-\mathbf{Q}(p, r, y)$

Also, considering (7), it is easy to conclude that for small values of $\delta p, \delta r$ and $\delta y$,

$\begin{aligned} \mathbf{Q}(\delta p, \delta r, \delta y)= & =\left[\begin{array}{ccc}1 & \delta y & -\delta p \\ -\delta y & 1 & \delta r \\ \delta p & -\delta r & 1\end{array}\right] \\ & =\left[\begin{array}{lll}1 & 0 & 0 \\ 0 & 1 & 0 \\ 0 & 0 & 1\end{array}\right]-\left[\begin{array}{ccc}0 & -\delta y & \delta p \\ \delta y & 0 & -\delta r \\ -\delta p & \delta r & 0\end{array}\right]=\mathbf{I}-\Psi\end{aligned}$

where $\mathbf{I}$ is an $3 \times 3$ identity matrix and $\Psi$ is the skew matrix of attitude error $\delta \vec{\varepsilon}$ defined in (2) and can be expressed as

$\delta \varepsilon=\left[\begin{array}{c}p-p_{I N S} \\ r-r_{I N S} \\ y-y_{I N S}\end{array}\right]$

Hence, $\delta \mathbf{Q}$ can be written as

$\delta \mathbf{Q}=(\mathbf{I}-\Psi) \mathbf{Q}-\mathbf{Q}=-\Psi \mathbf{Q}$

In (17), $\delta \vec{e}^{E N U}$ is proportional to the reciprocal of distance between the satellite and the receiver and negligible compared to the other term; the differential phase observation vector $\delta \vec{\varphi}$ can be calculated as

$\delta \vec{\varphi}=\vec{\varphi}_{G N S S}-\vec{\varphi}_{I N S}=-\frac{2 \pi}{\lambda} \mathbf{Z} \Psi \mathbf{Q} \vec{e}^{E N U}$ 
From the skew matrix properties,

$\delta \vec{\varphi}=-\frac{2 \pi}{\lambda} \mathbf{Z}\left(\operatorname{skew}\left(\mathbf{Q} \vec{e}^{B}\right)\right)^{T} \delta \vec{\varepsilon}=\frac{2 \pi}{\lambda} \mathbf{Z}\left(\operatorname{skew}\left(\mathbf{Q} \vec{e}^{B}\right)\right) \delta \vec{\varepsilon}$

The measurement model for pseudorange and pseudorange rate errors in (3) can be combined with the measured phase vectors of all of the satellites to create the following augmented measurement model: the ultra-tight GNSS/INS using collected signals by an actual antenna array in a harsh environment. For both tests, ultra-tight software receiver GSNRx ${ }^{\mathrm{TM}}$ is used as the major processing tool. The software, written in $\mathrm{C}++$, is capable of processing GPS/GLONASS/Galileo signal samples from one or more front ends (Petovello et al. 2008a, b).

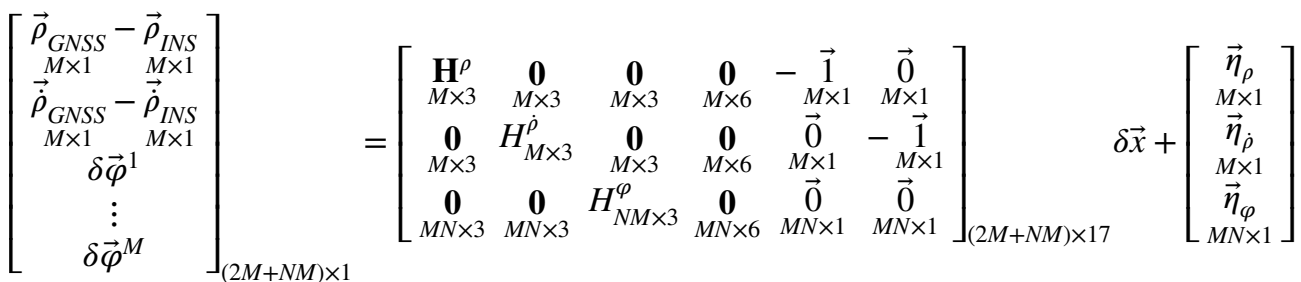

where $H_{N M \times 3}^{\varphi}$ is the design matrix formed from (25) and from all satellite signals. Another important advantage of GNSS/ INS/phased array integration is for the attitude parameter alignment process. East and north velocity measurements from accelerometers are conventionally used for leveling and initial estimation for pitch and roll angles. Heading alignment is performed by sensing the Earth's rotation rate from the gyroscope measurements in the up direction, known as gyro-compassing (Noureldin et al. 2013). Gyro-compassing is not accurate for low-grade IMUs. Moreover, the above methods require the receiver to be static. Attitude parameters estimated from array processing can be used as an external source that provides initial attitude parameters, especially the heading angle. Using an antenna array relaxes the static receiver condition for alignment process.

It should be also noted that in the presence of interference and antenna array uncertainties, the spatial phase measurement vector $\vec{\varphi}_{\text {GNSS }}$ in (15) is obtained after applying the calibration and projection matrices into the estimated steering vector as

$\vec{\varphi}_{\text {GNSS }}=$ phase $\left(\mathbf{C}^{-1} \mathbf{R} \vec{a}\right)$

The rest of the equations remains unchanged.

\section{Simulations and data analysis}

In this section, two data collection test results are presented. The first one focuses on testing the GNSS/INS/ distortionless beamformer integration proposed in the previous section and verifying its effectiveness in harsh environments using simulated array data in the absence of interference. The second test compares the performance of blind and distortionless beamformers integrated with

\section{Test one}

In this test, an array of GPS L1 C/A data was simulated using previously recorded 10-min GPS digital signals from a single antenna. Array signals were precisely simulated using the method outlined in (Vagle et al. 2016). The carrier Doppler, code delays and the navigation data bits extracted from processing the collected intermediate frequency (IF) samples were then used to generate array signals by employing the method of phase translations. Precise steering vectors are available for signal generation since exact locations of the satellites are known a priori and attitude parameters are available using a tactical IMU during the data collection. For this test, a dynamic user situation was considered. In order to evaluate the performance of the proposed approach in a harsh environment where the number of available satellite signals is limited due to blockage, the data generated include only the signals of four satellites, one of which is blocked every other $50 \mathrm{~s}$. An antenna array with three antenna elements in a linear configuration was used in the simulation. The data collection setup and environment is shown in Fig. 2. The NovAtel SPAN ${ }^{\mathrm{TM}}$ LCI system, which includes a NovAtel SPAN ${ }^{\circledR}$ enabled GNSS/INS receiver (SPAN SE) and a tactical grade IMU LCI, was used as reference. IMU measurements were sent to the receiver where a coupled GNSS/INS position, velocity and attitude solution were generated at a rate of $200 \mathrm{~Hz}$. Raw GPS data were also collected under line-of-sight (LOS) condition using another receiver as base station (fixed on a nearby building rooftop on the campus of the University of Calgary) to provide precise differential positioning. The raw IMU data were also used in the proposed GNSS/INS/distortionless beamformer integration.

In the first 100 and last $250 \mathrm{~s}$ of data collection, the antenna was static. As explained before, heading error 


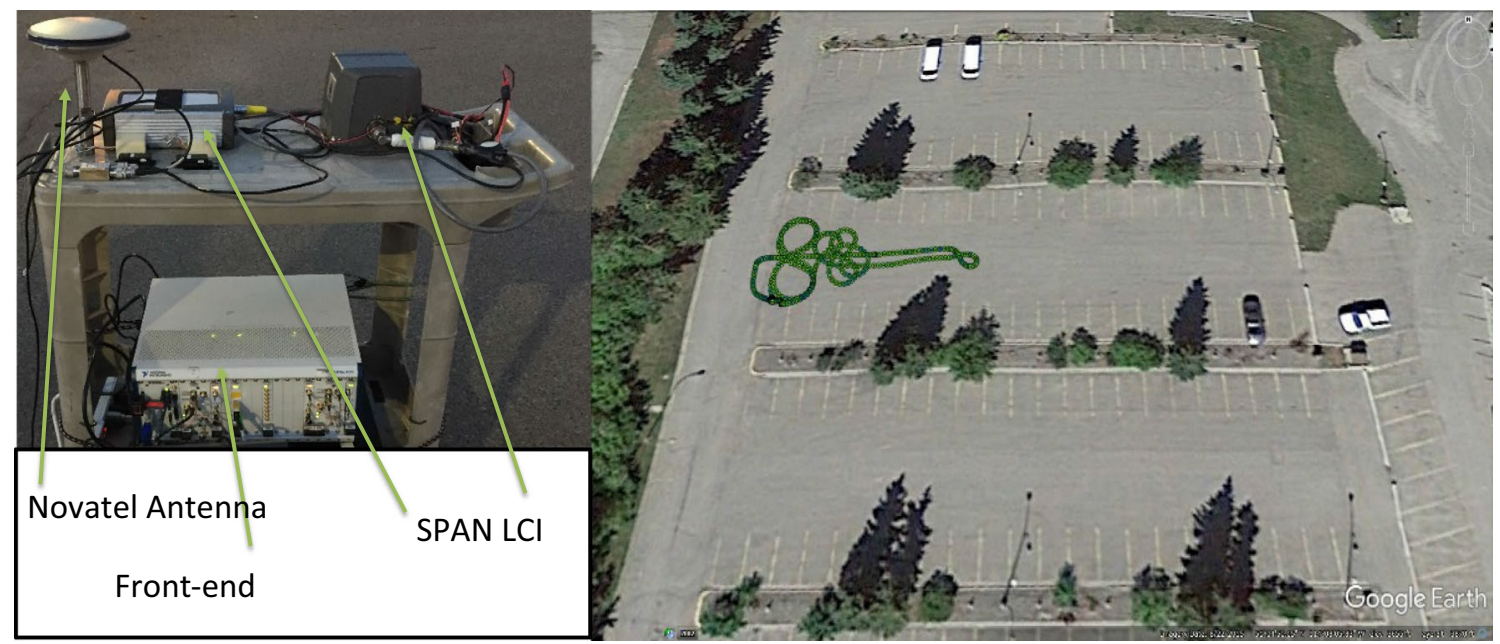

Fig. 2 Data collection setup and trajectory for the first test
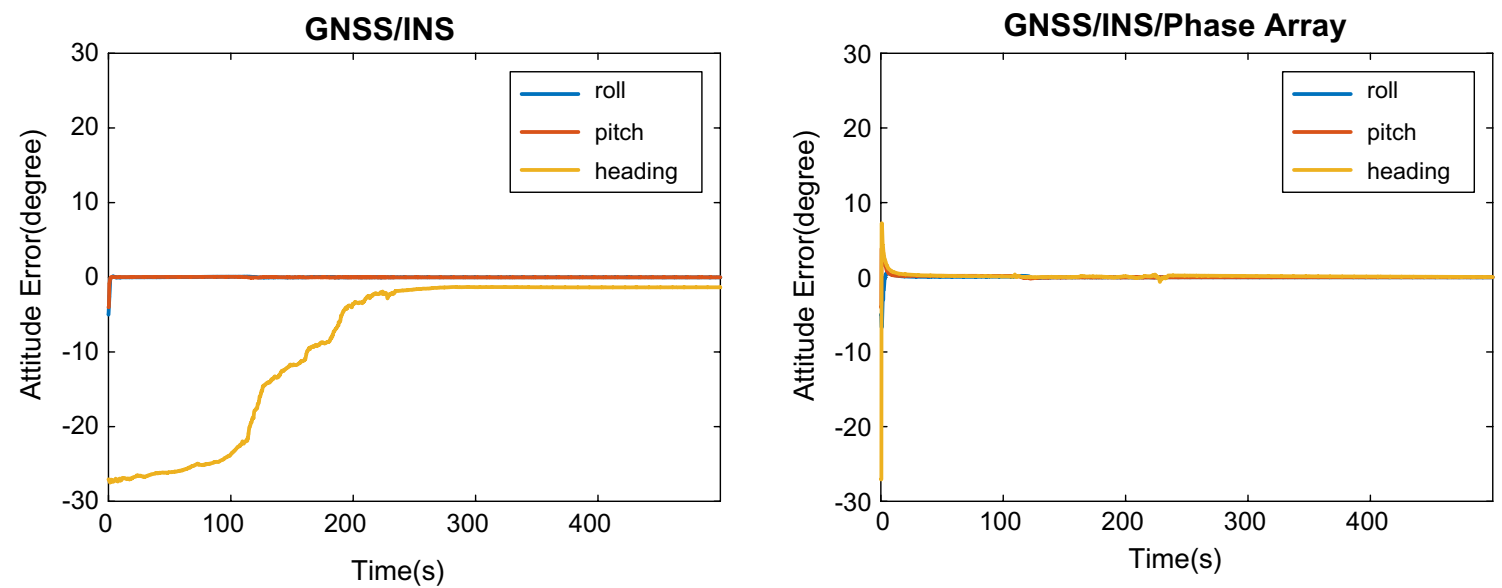

Fig. 3 Attitude error comparison between conventional GNSS/INSS receiver and the proposed GNSS/INS/phase array receiver

correction is challenging in a conventional GNSS/INS integration, while array processing is providing attitude parameters including heading. Figure 3 compares estimated heading angles using the conventional ultra-tight GNSS/INS integration with the proposed GNSS/INS/phased array integration approach when there is a $20^{\circ}$ initial heading error and $5^{\circ}$ initial roll and pitch errors. Using the proposed method, the initial heading error is corrected in less than a few seconds, while in the conventional approach, the error is slowly corrected after the receiver starts moving. As explained before, the north and east velocity component errors are bounded and also related to roll and pitch errors. Therefore, the velocity updates from the GNSS receiver can estimate and compensate roll and pitch errors very fast, but since there is no strong coupling with the heading error, it is not corrected over time especially for static cases. This in turn degrades the long-term accuracy of position and velocity estimates especially in harsh environments where GNSS may be regularly interrupted. Figure 3 shows that since the antenna is static at the end of the experiment, the heading error is not corrected and an offset remains.

ENU position and velocity errors for these two methods are also shown in Figs. 4 and 5. The array-based approach also increases the $\mathrm{C} / \mathrm{N}_{0}$ of each satellite signal. These improve the overall performance of the integrated system. Table 1 compares root-mean-square (RMS) and mean values of velocity, position and attitude errors between these two methods.

\section{Test two}

Figure 6 shows the test setup and data collection environment for this test. GPS L1 C/A signals were collected using an array of six NovAtel GPS Antennas (Model 501). The 

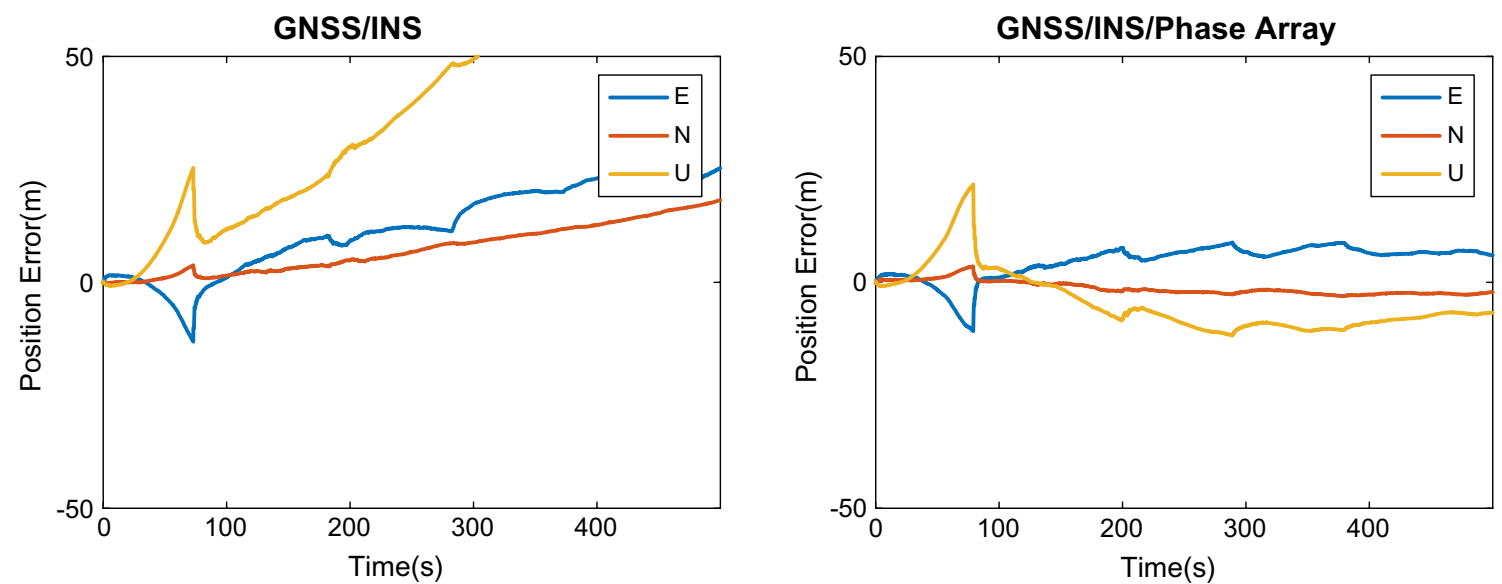

Fig. 4 ENU position error comparison between conventional GNSS/INSS receiver and the proposed GNSS/INS/phase array receiver
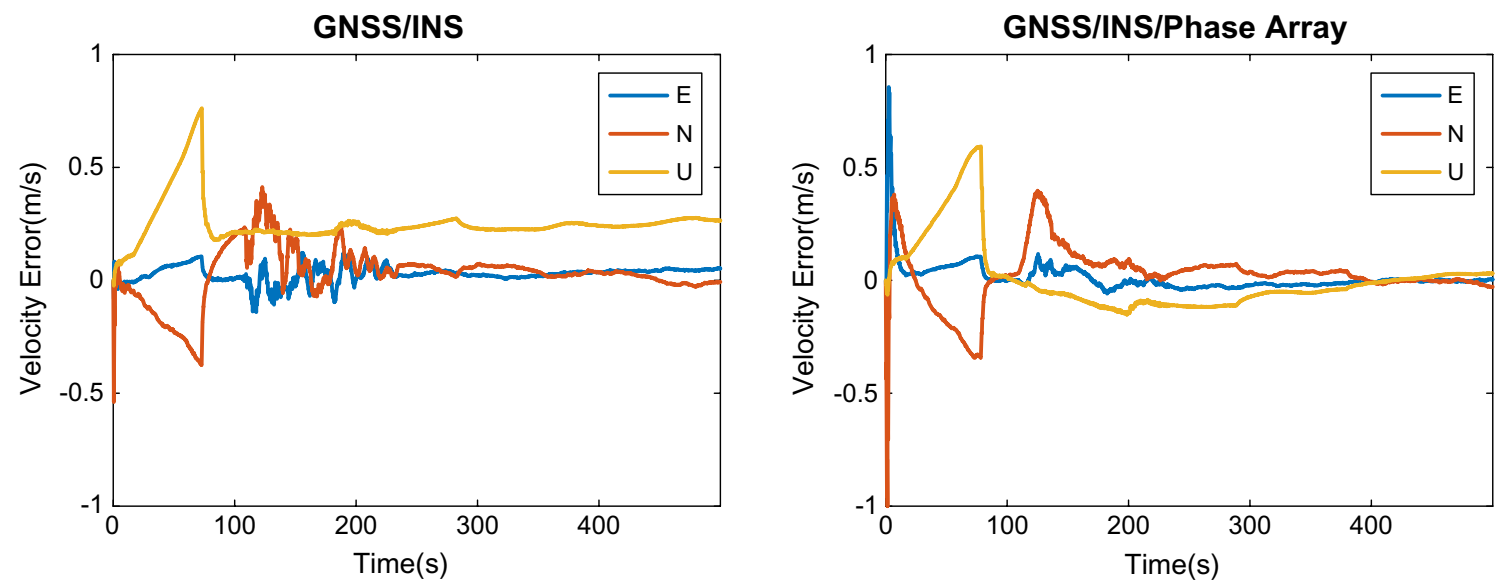

Fig. 5 ENU velocity error comparison between conventional GNSS/INSS receiver and the proposed GNSS/INS/phase array receiver

Table 1 ENU position and velocity and attitude RMS and mean error comparison between conventional GNSS/INSS receiver and the proposed GNSS/INS/phase array receiver

\begin{tabular}{|c|c|c|c|c|c|c|c|c|c|}
\hline $\begin{array}{l}\text { Method of } \\
\text { integration }\end{array}$ & E_Pos (m) & N_Pos (m) & U_Pos (m) & E_Vel (m/s) & N_Vel (m/s) & U_Vel (m/s) & Pitch $\left(^{\circ}\right)$ & Roll $\left(^{\circ}\right)$ & Heading $\left(^{\circ}\right)$ \\
\hline \multicolumn{10}{|l|}{ GNSS/INS } \\
\hline RMS & 15.5 & 9.1 & 49.6 & 0.04 & 0.12 & 0.27 & 0.18 & 0.13 & 13.19 \\
\hline Mean & 12.2 & 7.4 & 41.4 & 0.03 & 0.03 & 0.25 & -0.01 & 0.01 & -8.61 \\
\hline \multicolumn{10}{|c|}{ GNSS/INS/phased array } \\
\hline RMS & 6.0 & 1.9 & 8.1 & 0.07 & 0.13 & 0.15 & 0.34 & 0.28 & 0.96 \\
\hline Mean & 4.7 & -1.3 & -4.5 & 0.01 & 0.02 & 0.01 & 0.01 & 0.06 & 0.14 \\
\hline
\end{tabular}

antenna array was mounted on the top of a vehicle, and the six antenna elements were connected to phase coherent sixchannel Fraunhofer/TeleOrbit RF front ends. The sampling frequency was Fs $=20 \mathrm{MHz}$. The received signals were then down-converted, digitized and stored for post-processing. Similar to the previous data collection, the NovAtel SPAN
LCI system was used as a reference and also for providing raw IMU data. In this test, before testing the integrated system, the array was first calibrated in a parking lot with an open view to the satellites using the proposed method in (Daneshmand et al. 2014a, b). 


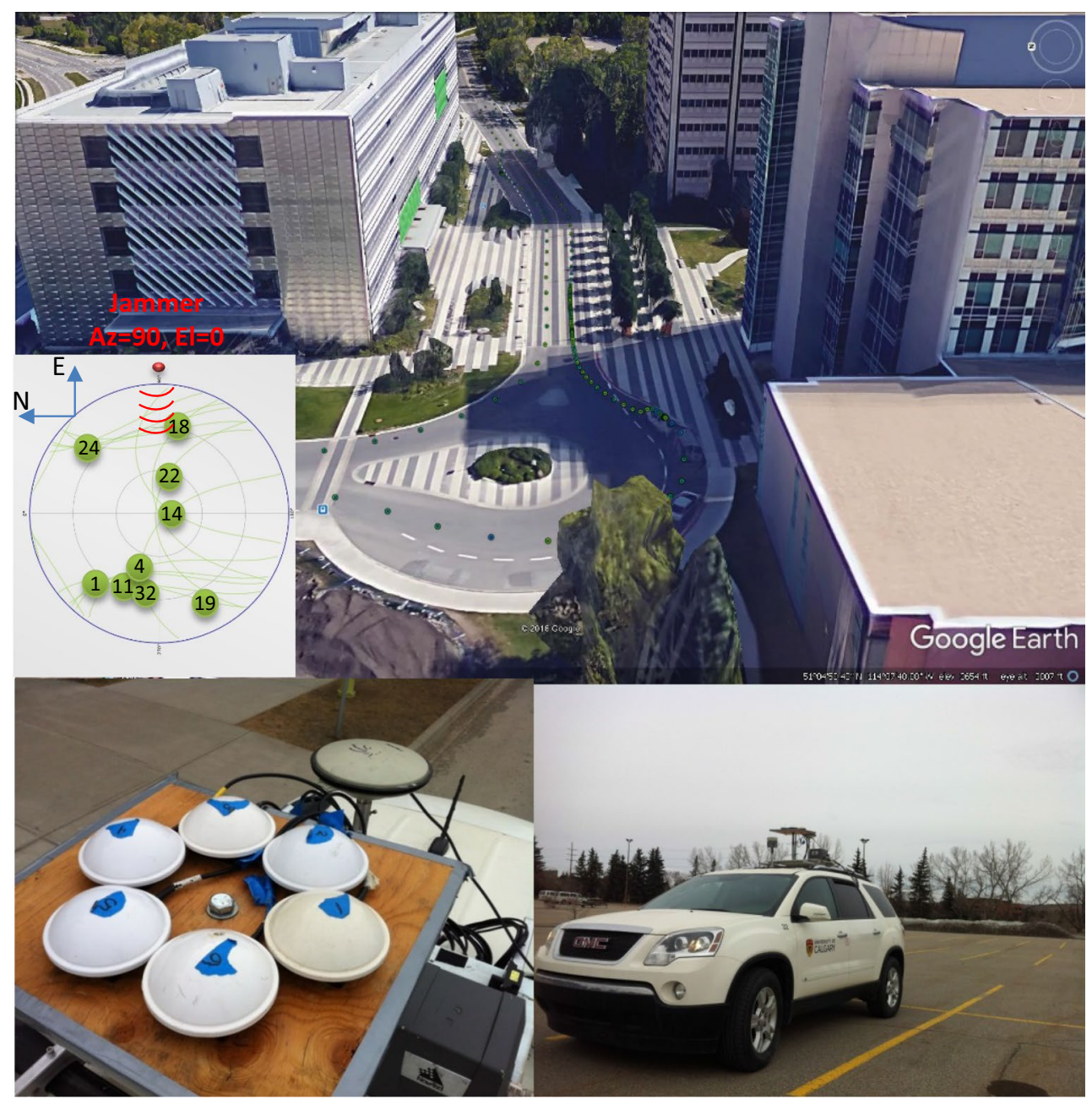

Fig. 6 Test setup and data collection environment for Test two
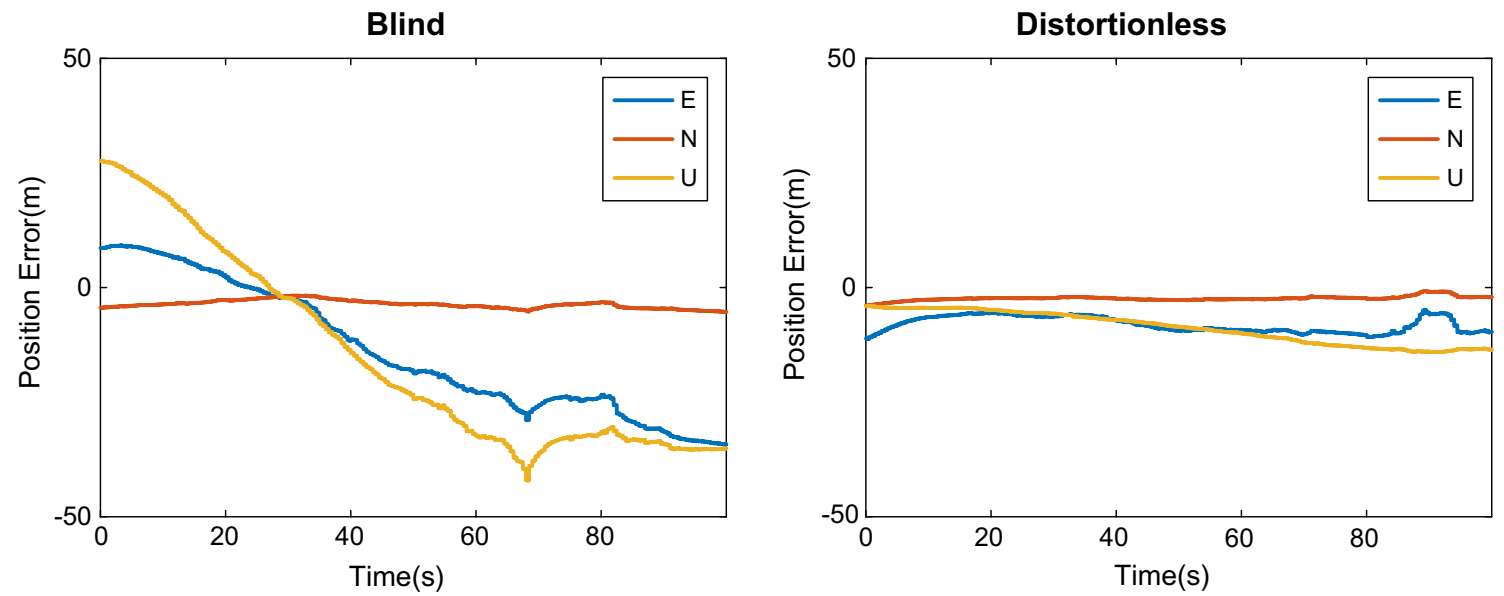

Fig. 7 ENU position error comparison between conventional GNSS/INS/blind beamformer and GNSS/INS/distortionless beamformer 

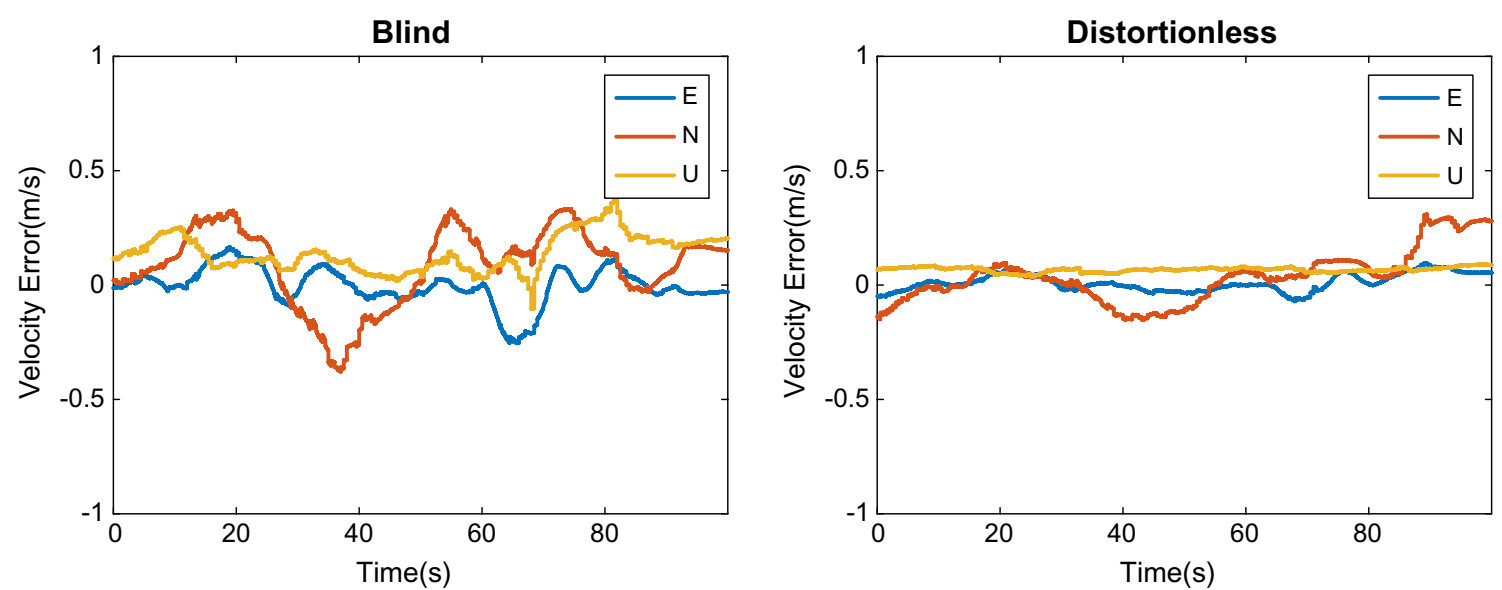

Fig. 8 ENU velocity error comparison between conventional GNSS/INS/blind beamformer and GNSS/INS/distortionless beamformer

Table 2 ENU position and velocity and attitude RMS and mean error comparison between GNSS/INS/blind beamformer and GNSS/INS/distortionless beamformer

\begin{tabular}{|c|c|c|c|c|c|c|c|c|c|}
\hline $\begin{array}{l}\text { INS/GNSS/beam- } \\
\text { forming method }\end{array}$ & E_Pos (m) & N_Pos (m) & U_Pos (m) & E_Vel $(\mathrm{m} / \mathrm{s})$ & N_Vel (m/s) & U_Vel (m/s) & Pitch $\left(^{\circ}\right)$ & Roll $\left(^{\circ}\right)$ & Heading $\left(^{\circ}\right)$ \\
\hline \multicolumn{10}{|l|}{ Blind } \\
\hline RMS & 19.8 & 3.7 & 25.9 & 0.08 & 0.19 & 0.08 & 0.03 & 0.03 & 3.29 \\
\hline Mean & -13.9 & -3.6 & -14.9 & -0.01 & 0.08 & 0.14 & 0.03 & 0.01 & -3.22 \\
\hline \multicolumn{10}{|c|}{ Distortionless beamformer } \\
\hline RMS & 8.2 & 2.4 & 9.5 & 0.04 & 0.12 & 0.07 & 0.03 & 0.04 & 1.25 \\
\hline Mean & -8.1 & -2.3 & -8.8 & 0.01 & 0.03 & 0.07 & 0.02 & 0.02 & -1.22 \\
\hline
\end{tabular}

As shown in Fig. 6, to create a harsh environment, a vehicle was driven between tall buildings where some satellite signals could be blocked and strong multipath reflections affected the received signals. Moreover, one interfering signal was added to the recorded data. In order to test and evaluate the performance of the proposed method under

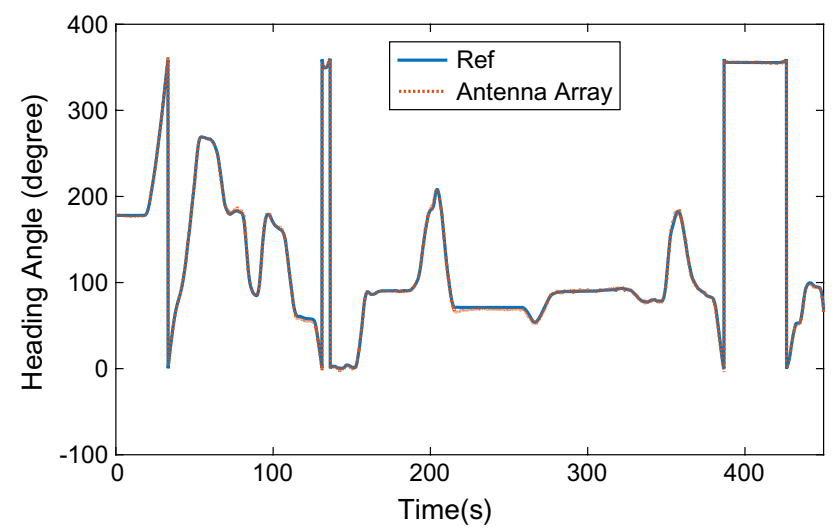

Fig. 9 Vehicle heading estimates using array GNSS-only processing interference, a continuous wave $(\mathrm{CW})$ interfering signal was generated using a software simulator and added to the recorded samples. The position of the interference does not change and is assumed to be located far enough so that its azimuth and elevation angles are constant during the 2-min duration of the data collection. Jammer interference-to-noise ratio (JINR) was $15 \mathrm{~dB}$, and its carrier frequency was at the L1 center frequency.

Errors in ENU coordinates for position and velocity are shown in Figs. 7 and 8. RMS and mean values of velocity, position and attitude errors between these two methods are shown in Table 2. As suggested in this table, the distortionless beamformer integrated with GNSS/INS outperforms the blind beamformer integrated with GNSS/INS and leads to the less RMS position, velocity and heading errors by a factor of 2-3. The factors contributing to this are extra attitude information from the array processing and avoidance of unintentional nulls for the GNSS signals. Moreover, steering a main lobe of beam pattern toward the direction of a satellite LOS signal not only increases the $\mathrm{C} / \mathrm{No}$ but also reduces the power of multipath reflections in the distortionless beamformer. In this analysis, the raw IMU data are 
Table 3 Mean and RMS values of pitch, roll and heading angle errors for array GNSS-only processing

\begin{tabular}{lllllllr}
\hline & \multicolumn{2}{l}{ First $150 \mathrm{~s}$ of data } & & \multicolumn{4}{l}{ Last 300 s of data } \\
\cline { 2 - 3 } & Pitch & Roll & Heading & & Pitch & Roll & Heading \\
\hline RMS error $\left(^{\circ}\right)$ & 0.99 & 0.74 & 1.47 & & 1.87 & 1.75 & 1.74 \\
mean error $\left(^{\circ}\right)$ & 0.03 & -0.45 & -0.04 & & 1.64 & -1.62 & -0.70 \\
\hline
\end{tabular}

obtained from the tactical grade IMU; however, the standard IMU calibration process has not been performed to better show how the errors are estimated and compensated in a harsh environment in these two methods. It should be also noted that the overall improvement is higher for lower-grade IMUs since the additional attitude information provided by array is independent of IMU measurements.

In order to evaluate the accuracy of the attitude updates from array processing, GNSS-only processing in the absence of interference was performed on the entire data, starting from open sky to a semi-urban area. The method is the extended Kalman filter described in (15)-(25) and the phase update of the design matrix in (26). The only difference is that the linearization is based on the best previous estimate instead of estimates from INS and IMU measurements. Figure 9 shows heading estimates using the proposed approach and the reference vehicle heading obtained from the SPAN LCI reference system. The satellite signals used for this analysis are the same as ones used in Test two. The RMS and mean errors during the first $150 \mathrm{~s}$ and the rest of data are shown separately in Table 3. Multipath is stronger in the second half when the vehicle drives between tall buildings, and therefore, the performance of attitude estimation is slightly degraded. As mentioned before, the accuracy of attitude estimates using array processing does not depend on IMU measurements. Therefore, the overall improvement due to array attitude updates in the integrated system would be higher for lower-grade IMUs with significant errors that need to be corrected over time.

\section{Conclusions}

Different types of GNSS/INS integration with blind and distortionless beamformers were studied. The focus was on ultra-tight and tightly coupled GNSS/INS/distortionless beamformer, which not only has all the advantages of antenna array processing and INS in dealing with blockage, severe multipath and jamming, but also provides attitude updates from array GNSS signals. The structure of a receiver and modifications to the conventional Kalman filter for this implementation was described. Two sets of data collection and analysis were proposed to verify the applicability of the proposed integrated system and to evaluate and compare its performance with the conventional methods for both noninterference and interference environments.

Open Access This article is distributed under the terms of the Creative Commons Attribution 4.0 International License (http://creativecommons.org/licenses/by/4.0/), which permits unrestricted use, distribution, and reproduction in any medium, provided you give appropriate credit to the original author(s) and the source, provide a link to the Creative Commons license, and indicate if changes were made.

\section{References}

Arribas J, Fernandez-Prades C, Closas P (2013) Multi-antenna' techniques for interference mitigation in GNSS signal acquisition. EURASIP J Adv Signal Process 1:143-152

Backen S, Akos DM, Nordenvaad ML (2008) Post-processing dynamic GNSS antenna array calibration and deterministic beamforming. In: Proceedings of ION GNSS 2008, Institute of Navigation, Savannah, GA, USA, 16-19 September 2008, pp 2806-2814

Borio D, Dovis F, Kuusniemi H, Presti LL (2016) Impact and detection of GNSS jammers on consumer grade satellite navigation receivers. Proc IEEE 104(6):1233-1245

Brown A, Matthews B (2006) A robust GPS/INS kinematic integrity algorithm for aircraft landing. In: Proceedings of ION GNSS 2006, Institute of Navigation, Fort Worth, TX, USA, 26-29 September 2006, pp 715-725

Carrie G, Vincent F, Deloues T, Pietin D, Renard A (2005) A new blind adaptive antenna array for GNSS interference cancellation. In: Proceedings of the conference record of the thirty-ninth Asilomar conference on signals, systems and computers, Pacific Grove, CA, USA, 28 October-1 November 2005, pp 1326-1330

Citron TK, Kailath T (1984) An improved eigenvector beamformer. In: IEEE international conference on ICASSP 84 acoustics, speech, and signal processing, 19-21 March 1984, San Diego CA, pp 718-721

Cuntz M, Konovaltsev A, Meurer M (2016) Concepts, development, and validation of Multiantenna GNSS receivers for resilient navigation. Proc IEEE 104(6):1288-1301

Daneshmand S, Broumandan A, Sokhandan N, Lachapelle G (2013) GNSS multipath mitigation with a moving antenna array. IEEE Trans Aerosp Electron Syst 49(1):693-698

Daneshmand S, Sokhandan N, Lachapelle G (2014a) Precise GNSS attitude determination based on antenna array processing. In: Proceedings of ION GNSS 2014, Institute of Navigation, Tampa, FL, USA, 8-12 September 2014, pp 2555-2562

Daneshmand S, Sokhandan N, Zaeri-Amirani M, Lachapelle G (2014b) Precise calibration of a GNSS antenna array for adaptive beamforming applications. Sensors 14(6):9669-9691

Daneshmand S, Marathe T, Lachapelle G (2016) Millimetre level accuracy GNSS positioning with the blind adaptive beamforming method in interference environments. Sensors 16(11):1824. https://doi.org/10.3390/s16111824 
De Lorenzo DS, Gautier J, Rife J, Enge P, Akos D (2005) Adaptive array processing for GPS interference rejection. In: Proceedings of ION GNSS 2005, Institute of Navigation, Long Beach, CA, USA, 13-16 September 2005, pp 618-627

Fante R, Vaccaro JJ (2000) Wideband cancellation of interference in a GPS receive array. IEEE Trans Aerosp Electron Syst 36(2):549-564

Fernández-Prades C, Arribas J, Closas P (2016) Robust GNSS receivers by array signal processing: theory and implementation. Proc IEEE 104(6):1207-1220

Godha S, Lachapelle G, Cannon ME (2006) Integrated GPS/INS system for pedestrian navigation in a signal degraded environment. In: Proceedings of ION GNSS 2006, Institute of Navigation, Fort Worth, TX, USA, 26-29 September 2006, pp 2151-2164

Grewal MS, Weill LR, Andrews AP (2007) Global positioning systems, inertial navigation, and integration, 2nd edn. Wiley-Interscience, Hoboken

Groves PD (2008) Principles of GNSS, inertial and multi-sensor integrated navigation systems. Artech House, Boston

Kim US, De Lorenzo DS, Gautier J, Enge P, Orr JA (2004) Phase effects analysis of patch antenna CRPAs for JPALS. In: Proceedings of ION GNSS 2004, Institute of Navigation, Long Beach, CA, USA, 21-24 September 2004, pp 1531-1538

Konovaltsev A, Cuntz M, Haettich C, Meurer M (2013) Performance analysis of joint multi-antenna spoofing detection and attitude estimation. In: Proceedings of ION ITM 2013, Institute of Navigation, San Diego, CA, USA, 27-29 January 2013, pp 864-872

Li M, Dempster AG, Balaei AT, Rizos C, Wang F (2011) Switchable beam steering/null steering algorithm for $\mathrm{CW}$ interference mitigation in GPS C/A code receivers. IEEE Trans Aerosp Electron Syst 47(3):1564-1579

Li Q, Wang W, Xu DJ, Wang XP (2014) A robust anti-jamming navigation receiver with antenna array and GPS/SINS. IEEE Commun Lett 18:467-470

Noureldin A, Karamat TB, Eberts MD, El-Shafie A (2009) Performance enhancement of MEMS based INS/GPS integration for low cost navigation applications. IEEE Trans Veh Technol 58(3):1077-1096

Noureldin A, Karamat TB, Georgy J (2013) Fundamentals of inertial navigation, satellite-based positioning and their integration. Springer, Berlin

Petovello MG, Lachapelle G (2006) Comparison of vector-based software receiver implementations with application to ultra-tight GPS/ INS integration. In: Proceedings of ION GNSS 2006, Institute of Navigation, Fort Worth, TX, USA, 26-29 September 2006, pp 1790-1799

Petovello MG, O’Driscoll C, Lachapelle G, Borio D, Murtaza H (2008a) Architecture and benefits of an advanced GNSS software receiver. J Glob Position Syst 7(2):156-168

Petovello MG, O'Driscoll C, Lachapelle G (2008b) Carrier phase tracking of weak signals using different receiver architectures. In: Proceedings of ION NTM 2008, Institute of Navigation, San Diego, CA, USA, 28-30 January 2008, pp 781-791

Petovello MG, O’Keefe K, Lachapelle G, Cannon ME (2009) Measuring aircraft carrier flexure in support of autonomous aircraft landings. IEEE Trans Aerosp Electron Syst 42(2):523-535
Reed CW, Yao K (1998) Performance of blind beamforming algorithms. In: Proceedings of the ninth IEEE SP Workshop on statistical signal and array processing, Portland, OR, USA, 14-16 September 1998, pp 256-259

Seco-Granados GJ, Fernández-Rubio A, Fernández-Prades C (2003) ML estimator and hybrid beamformer for multipath and interference mitigation in GNSS receivers. IEEE Trans Signal Process 53(3):1194-1208

Sgammini M, Antreich F, Kurz L, Meurer M, Noll TG (2012) Blind adaptive beamformer based on orthogonal projections for GNSS. In: Proceedings of ION GNSS 2012, Institute of Navigation, Nashville, TN, USA, 17-21 September 2012, pp 926-935

Vagle N, Broumandan A, Jahromi AJ, Lachapelle G (2016) Performance analysis of GNSS multipath mitigation using antenna arrays. J Glob Position Syst 14(2):1-15

Van Trees HL (2002) Optimum array processing, detection, estimation, and modulation theory. Part IV. Wiley Interscience, New York

Wong K, Schwarz P, Cannon ME (1988) High-accuracy kinematic positioning by GPS-INS. Navigation 35(2):275-287

Zoltowski MD, Gecan AS (1995) Advanced adaptive null steering concepts for GPS. In: Proceedings of the 1995 IEEE Conference Record, Military Communications Conference (MILCOM'95), San Diego, CA, USA, 5-8 November 1995, pp 1214-1218

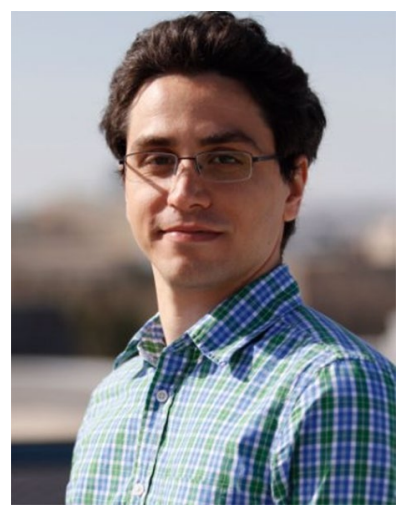

Saeed Daneshmand received his B.Sc. in Electrical engineering from the IUST University, in 2006, and M.Sc. in Telecommunication engineering from the Amirkabir University of Technology, in 2009. He obtained his Ph.D. in Geomatics engineering from the University of Calgary, in 2013. He worked for 4 years as a senior research associate with the PLAN Group in the University of Calgary before he started his career in a GNSS industry.

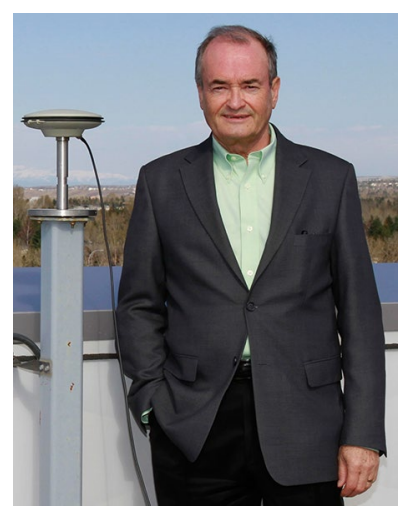

Gérard Lachapelle is Professor Emeritus at the University of Calgary, which he joined in 1988. He has been involved in a multitude of global navigation satellite system (GNSS) R\&D projects since 1980 , ranging from RTK positioning to indoor location and GNSS signal processing enhancements. 